\title{
Yaw Moment Compensation for Humanoid Robot via Arms Swinging
}

\author{
Liang Yang* and Chunjian Deng
} School of Computer Engineering, University of Electronic Science and Technology of China Zhongshan Institute,
Zhongshan, Guangdong province, 528402, China

\begin{abstract}
This paper investigates the problems of the z-axis dynamic balance and high energy consumption in practical application of humanoid robot. Considering z-axis dynamic balance conditions, a novel yaw moment compensation solution based on arms-swinging is proposed by computing the optimal arms-swinging trajectories to counteract the yaw moment caused by the movement of humanoid robot. Given the optimized arms-swinging motion, an adaptive control system is proposed to track the desired trajectories with model uncertainties. From the Lyapunov stability analysis, the adaptation law is induced and the uniformly boundness of all signals in a closed-loop adaptive system is proved. Simulation results validate the proposed method.
\end{abstract}

Keywords: Yaw Moment, Humanoid Robot, Arms Swinging, Moment Compensation.

\section{INTRODUCTION}

The stability of humanoid walking is one of the fundamental problems, which impacts the application of humanoid robot. Many researchers have proposed control methods based on ZMP criterion, which has been taken as the most commonly used stability criterion for humanoid locomotion [1]. ZMP is defined as that point on the ground at which the net moment of the inertial forces and the gravity forces has no component along the horizontal axis [2-4]. According to the definition, ZMP stability criteria can only guarantee the moment balance in horizontal axis, but not for vertical axis. Here, the vertical moment component with respect to ZMP is named yaw moment, which is caused by the movement of humanoid robot. Usually, it is the ground reaction moment to balance the undesired yaw moment via the contact between foot and the ground. The deviation from the desired trajectory would happen when the yaw moment exceeds the maximum ground reaction moment.

Therefore, it is necessary to compensate yaw moment for humanoid robot to guarantee the stable walking. A bunch of methods on how to compensate yaw moment have been proposed. $\mathrm{Yu}$ [5] analyzed the relationship between yaw moment and waist rotation, and proposed a method to generate waist rotation trajectories which counteract the undesired yaw moment. Following the similar strategy, Ugurlu [6] utilized Eulerian ZMP resolution to compute the yaw moment and exerts a secondary moment to counteract yaw moment via waist rotation. This method considers the influence caused by intrinsic angular momentum rate changes and has a good performance. However, counteracting yaw moment by rotating waist joint needs more energy and the gait is usually unnatural and can't guarantee the dynamic balance in vertical axis.

Arms swing is considered as a good choice to compensate the yaw moment due to the fact that this action is quite natural and can be observed during human walking [7]. Xing [1] proposed a method to generate arms-swinging trajectories which help to counterbalance the impact caused by the yaw moment. $\mathrm{Fu}$ [8] optimized the angle parameters of arms swinging and get arms angle trajectories of arms swinging based on cubic spline interpolation. All these methods appear to be helpful in handling the undesired yaw moment problem. However, the off-the-shelf techniques to generate arms-swinging trajectories don't have a consideration about energy consumption, which limits large-scale application of humanoid robot in a way. Wang [9] proposed systematic control based ZMP criterion, which reduce the energy consumption. But this method doesn't consider the impact caused by the yaw moment.

To address this problem, a novel yaw moment control based on arms-swinging is proposed. By designing energyefficiency optimized arms-swinging trajectories, the proposed method provides remarkable descent rate of energy consumption. Furthermore, to obtain a better tracking performance, an adaptive controller is proposed and the analysis on system stability is provided.

\section{PROBLEM STATE}

ZMP is one of the most popular stability criteria for humanoid robot. But ZMP stability criterion only consider the moment balance in the horizontal direction, which couldn't guarantee the vertical balance. During humanoid walking, arms, swing leg and trunk move forward in different plane, which inevitably produce a moment with respect to the 


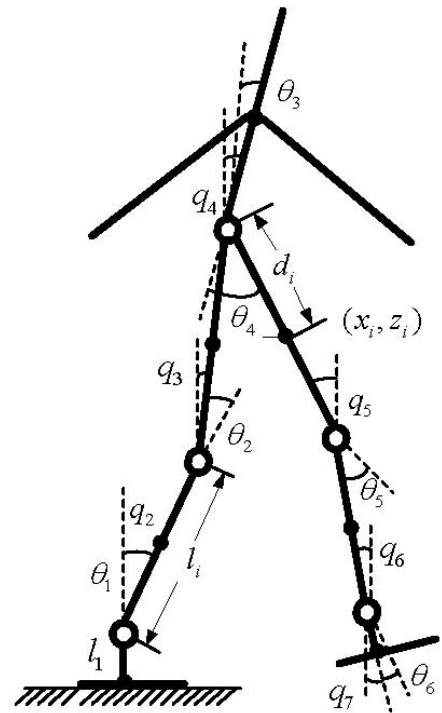

Fig. (1). Model Saggital view of link model for humanoid robot.

support foot and influence the dynamic balance in vertical axis. Thus it is necessary to analyze the moment balance in vertical axis.

\subsection{Influence Analysis of Yaw Moment on Stability}

Hirabayashi [10] analyzed the stability influence caused by yaw moment based on 3D inverted pendulum model. While this model is a simple system with a point mass and a massless telescopic leg, which couldn't describe all the physical characters of humanoid robot. In order to analyze the influence on stability caused by yaw moment, we take thigh, leg, arm and foot as homogeneous connective links and setup connective links model as shown in Fig. (1).

Based on connective links model, the expression of yaw moment is defined as below, which ignores the effect of inertia moment.

$M_{\text {total }}^{z}=\sum_{i=1}^{n} m_{i} \ddot{y}_{i}\left(x_{i}-x_{\mathrm{zmp}}\right)-\sum_{i=1}^{n} m_{i} \ddot{x}_{i}\left(y_{i}-y_{\mathrm{zmp}}\right)$

Where $M_{\text {total }}^{z}$ is representing the vertical component of the total moment which is acted on ZMP by the rest system except the support foot. $\left(x_{z m p}, y_{z m p}\right)$ is representing the coordinate of the zero moment point. $n=1,2, \ldots, 9$. And the ZMP coordinates can be obtained via the following equation.

$$
\left\{\begin{array}{l}
x_{\mathrm{zmp}}=\frac{\sum_{i=1}^{n}\left[m_{i}\left(\ddot{z}_{i}+g\right) x_{i}-m_{i} \ddot{x}_{i} z_{i}\right]}{\sum_{i=1}^{n} m_{i}\left(\ddot{z}_{i}+g\right)} \\
y_{\mathrm{zmp}}=\frac{\sum_{i=1}^{n}\left[m_{i}\left(\ddot{z}_{i}+g\right) y_{i}-m_{i} \ddot{x}_{i} z_{i}\right]}{\sum_{i=1}^{n} m_{i}\left(\ddot{z}_{i}+g\right)}
\end{array}\right.
$$

where $x_{i}, y_{i}$ and $z_{i}$ represent the coordinates of the $i$ th link, and $g$ is gravity force. One dot and two dots represent first and second derivatives with respect to time.

As widely known, the support foot sole is the only contact with the ground during walking. The ground reaction $R=\left(R_{x}, R_{y}, R_{z}\right)$ is the only external force to balance yaw moment. In order to balance the yaw moment, the following inequality should hold on.

$$
\left|M_{R}^{z}\right| \geq\left|M_{\text {total }}^{z}\right|
$$

where $\$ M \_R^{\wedge} \mathrm{z} \$$ is the $\mathrm{z}$-axis moment generated by the $\mathrm{z}$ axis component $R_{z}$ of the ground reaction $R$.

\subsection{The Performance of Yaw Moment Control}

In order to estimate the energy consumption for humanoid robot during walking, the average mechanical power $P_{\text {average }}$ is given, which is shown as below.

$$
P_{\text {average }}=\frac{1}{N} \sum_{i=1}^{n} \sum_{j=1}^{N}\left|\tau_{i}(j) \dot{q}_{i}(j)\right|
$$

where is the sum of all joints, $N$ is the total sampling time, $\tau_{i}(j)$ represents the output torque of $i$ th joint at $j$ th sampling moment.

The yaw stability margin is used to measure the stability of $\mathrm{z}$ axis, which is expressed as the equation (5)

$$
\begin{aligned}
& M_{\text {yaw }}^{\text {margin }}=\left|M_{R}^{z}\right|-\left|M_{\text {total }}^{z}\right| \\
& M_{\text {total }}^{z}=M_{A}^{z}+M_{z}
\end{aligned}
$$

Where $M_{R}^{z}$ represents the vertical component of the ground reaction moment and $M_{\text {total }}^{z}$ is the total yaw moment during walking. $M_{A}^{z}$ is the moment generated by armsswinging and $M_{z}$ represents the moment generated by the movement of body and legs.

\section{YAW MOMENT COMPENSATION BASED ON ARMS SWINGING}

Control method based on arms swinging is proposed to obtain the trade-off between dynamic balance and energy efficiency by minimizing the amplitude of arm-swinging while guaranteeing the $\mathrm{z}$-axis moment balance. There are three parts in the control system: gait planning, internal-loop controller of joints angle trajectories and outer-loop armswinging optimization control. The system diagram is shown in Fig. (2). Arms swinging controller get the optimized arm swinging angle acceleration according to the $q_{r}, \dot{q}_{r}, \ddot{q}_{r}$, and adaptive controller is responsible for tracking the armsswinging angles trajectories. 


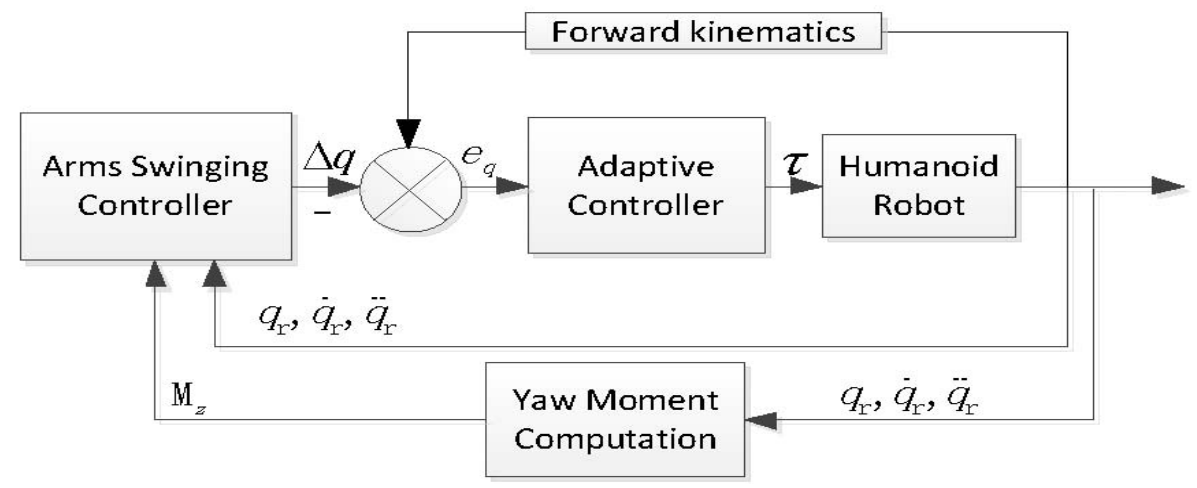

Fig. (2). Model Saggital view of link model for humanoid robot.

\subsection{Dynamic Balance Optimization}

According to $\mathrm{Fu}$ [8], the moment generated by arms swinging can be expressed as follows:

$M_{a}=m_{a} W_{a} l_{a}\left(\dot{q}_{a}^{2} \sin \left(q_{a}\right)-\ddot{q}_{a} \cos \left(q_{a}\right)\right) / 2$

Where $m_{a}$ is the mass of arm, $l_{a}$ is the length of arm and $q_{a}$ represents intersection angle between arm and vertical direction as shown in Fig. (3).

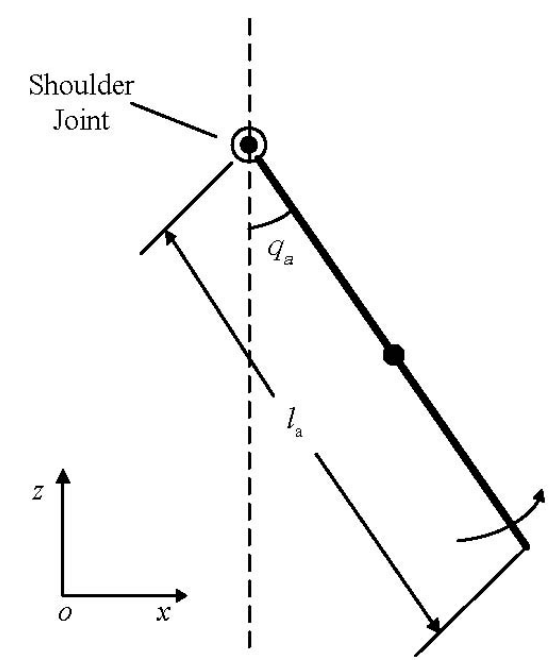

Fig. (3). The schematic diagram of arm swinging.

In order to compensating of yaw moment via arms swinging with high energy efficiency, the dynamic optimization problem could be modeled as the equation (8).

$$
\left\{\begin{array}{cc}
\min & J(f)=\frac{1}{2} \ddot{q}_{a}{ }^{T} Q \ddot{q}_{a}+b^{T} \ddot{q}_{a} \\
\text { s.t. } & M_{a}=M_{z}
\end{array}\right.
$$

where $Q$ is the symmetric and positive definite matrix with appropriate dimension. The above function $J(f)$ represents the minimum weighted norm of arms swinging angles acceleration when $b=0$.

Let $b=0$ and substitute the equation (7) to equation (9) results in

$$
\left\{\begin{aligned}
\min \quad J(f) & =\frac{1}{2} \ddot{q}_{a}^{T} Q \ddot{q}_{a} \\
\text { s.t. } \quad \cos \left(q_{a}\right) \ddot{q}_{a} & =\sin \left(q_{a}\right) \dot{q}_{a}-d
\end{aligned}\right.
$$

where $d=\frac{2 M_{z}}{m_{a} W_{a} l_{a}}$. The problem of arms-swinging control has been successfully transferred to a quadratic programming problem. This paper use Lagrange multiplier method to solve the quadratic programming problem.

Let $Q$ be identity matrix and suppose the following equation holds

$H\left(\ddot{q}_{a}\right)=\frac{1}{2} \ddot{q}_{a}^{2}+\lambda\left(\cos \left(q_{a}\right) \ddot{q}_{a}-\sin \left(q_{a}\right) \dot{q}_{a}+d\right)$

According to the Lagrange Multiplier Rule, the following equation holds.

$\frac{\partial H\left(\ddot{q}_{a}\right)}{\partial \ddot{q}_{a}}=0$

$\frac{\partial H\left(\ddot{q}_{a}\right)}{\partial \ddot{\lambda}}=0$

Substituting equation (10) to equation (11)-(12), the following equation are obtained.

$\ddot{q}_{a}=\frac{\sin \left(q_{a}\right) \dot{q}_{a}-d}{\cos \left(q_{a}\right)}$

where the arm-swinging angle velocity is obtained via the following equation.

$\dot{q}_{a}(n)=q_{a}(n)-q_{a}(n-1)$ 
Table 1. The configuration parameters of humanoid robot.

\begin{tabular}{|c|c|c|c|c|c|}
\hline & Trunk & Arm & Thigh & Shank & Foot \\
\hline \hline Length(m) & 0.28 & 0.25 & 0.14 & 0.11 & 0.04 \\
\hline Mass(kg) & 1.38 & 0.423 & 0.533 & 0.423 & 0.20 \\
\hline
\end{tabular}

Where $\mathrm{n}$ is sampling time, The equation (13) holds when $q_{a} \neq \frac{\pi}{2},-\frac{\pi}{2}, \pi,-\pi$ in the physical range of arm-swinging angle.

\subsection{The Design of Adaptive Controller}

In order to improve the tracking performance and reduce the impact caused by model uncertainties, an adaptive controller based on arms swinging is proposed and the stability of the controller has been proved. According to the Lagrangian approach, the dynamics equation can be expressed as follows:

$$
\tau=\mathbf{M}(q) \ddot{q}+\mathbf{C}(q, \dot{q}) \dot{q}+\mathbf{G}(q)+\tilde{\varphi}
$$

where $\mathrm{M}(q)$ is the positive definite inertial matrix; $\mathrm{C}(q, \dot{q})$ is the Coriolis and centrifugal matrix; $\mathrm{G}(q)$ is the gravitational force vector; $\tilde{\varphi}$ is the nonlinear term. The reference joint angle, reference joint velocity and residual error to reference joint velocity are defined as:

$\dot{q}_{\mathrm{r}}=\dot{q}_{\mathrm{d}}-\alpha \tilde{q}$

$\ddot{q}_{\mathrm{r}}=\ddot{q}_{\mathrm{d}}-\alpha \dot{\tilde{q}}$

$s=\dot{q}-\dot{q}_{r}$

where $\alpha$ is a positive constant. The reference angle acceleration velocity is obtained via the derivation of equation (18).

$\dot{s}=\ddot{q}-\ddot{q}_{r}$

Therefore, the adaptive control law is defined as

$$
\boldsymbol{\tau}=\mathbf{M}(q) \ddot{q}_{\mathrm{r}}+\mathbf{C}(q, \dot{q}) \dot{q}_{\mathrm{r}}+\mathbf{G}(q)-\mathbf{K}_{c} s
$$

where $K_{c}$ is a positive definite matrices. Substituting the controller equation (20) to the dynamics equation(15) results in

$$
\mathbf{M}(q) \dot{s}+\left(\mathbf{C}(q, \dot{q})+K_{s}\right) s+\tilde{\varphi}=0
$$

Considering the following Lyapunov function candidate

$$
V=\frac{1}{2} s^{\mathrm{T}} \mathbf{M}(q) s+\frac{1}{2} \tilde{\varphi}^{\mathrm{T}} \mathbf{K}_{c} \tilde{\varphi}
$$

The derivative of the equation (22) leads to
$\dot{V}=\frac{1}{2} s^{\mathrm{T}} \dot{\mathbf{M}}(q) s+s^{\mathrm{T}} \mathbf{M}(q) \dot{s}+\tilde{\varphi}^{\mathrm{T}} \mathbf{K}_{c} \dot{\tilde{\varphi}}$

Multiplying both sides of the equation(21) by $s^{\mathrm{T}}$ and get the following expression.

$\dot{V}=-s^{\mathrm{T}} \mathbf{K}_{c} \dot{s}-s^{\mathrm{T}} \mathbf{K}_{c}+\tilde{\varphi}^{\mathrm{T}} \mathbf{K}_{s} \dot{\tilde{\varphi}}$

According to the equation $\widetilde{\phi}=\hat{\phi}-\phi$, the following equation is obtained

$\dot{\widetilde{\phi}}=\dot{\hat{\phi}}$

Therefore, the parameter adaptive control law is defined as

$\dot{\hat{\varphi}}=\mathbf{K}_{c}^{-1} s^{\mathrm{T}}$

Substituting the equation (24)-(25) to the equation(24) results in

$\dot{V}=-s^{\mathrm{T}} \mathbf{K}_{c} \dot{s}-s^{\mathrm{T}} \tilde{\varphi}-\tilde{\varphi}^{\mathrm{T}} \mathbf{K}_{c} \dot{\hat{\varphi}}=-s^{\mathrm{T}} \mathbf{K}_{c} \dot{s}-s^{\mathrm{T}} \tilde{\boldsymbol{\varphi}}+\tilde{\varphi}^{\mathrm{T}} s$

$s^{\mathrm{T}} \tilde{\varphi}$ is a constant, $K_{c}$ is a positive matrix and the equation $s^{\mathrm{T}} \tilde{\varphi}=\tilde{\varphi}^{\mathrm{T}} s$ holds. Then the inequality $\dot{V}<0$ holds. So the system is stable. As $\dot{V}$ is uniformly continuous. According to Barbalat theorem [12], the system is asymptotically stable.

\section{SIMULATION AND RESULT ANALYSIS}

In this section, we first provide a brief presentation regarding the simulation environment and provide the examples of simulations to test our proposed method and the configuration parameters of humanoid is shown in Table 1.

In order to simulate all the walking phrase, three kinds of waling gaits are provided, which are start gait, periodic gait gait and stop gait. In the experiments, the first step is start gait, the second and third steps are period walking gait and the forth step is stop gait. Both left and right leg move forward one step is called one step period, which lasts 1.2 second. And the step length is $0.1 \mathrm{~m}$, sampling interval is 0.01 second.

In Matlab 7.11 simulation environment, the 2D and 3D schematic diagrams of humanoid walking are shown as Figs. (4 and 5) using the proposed arms-swinging method, which 


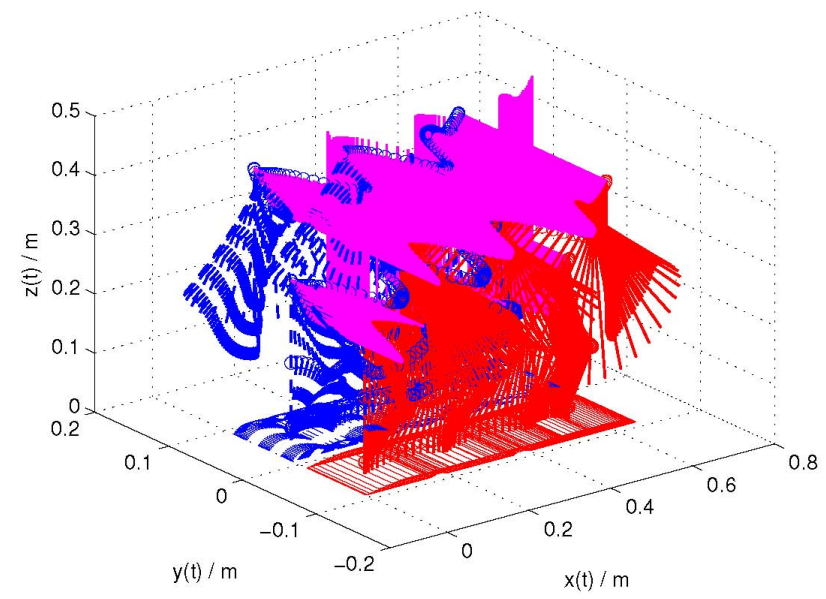

Fig. (4). The 3D schematic diagram of walking gait for humanoid robot.

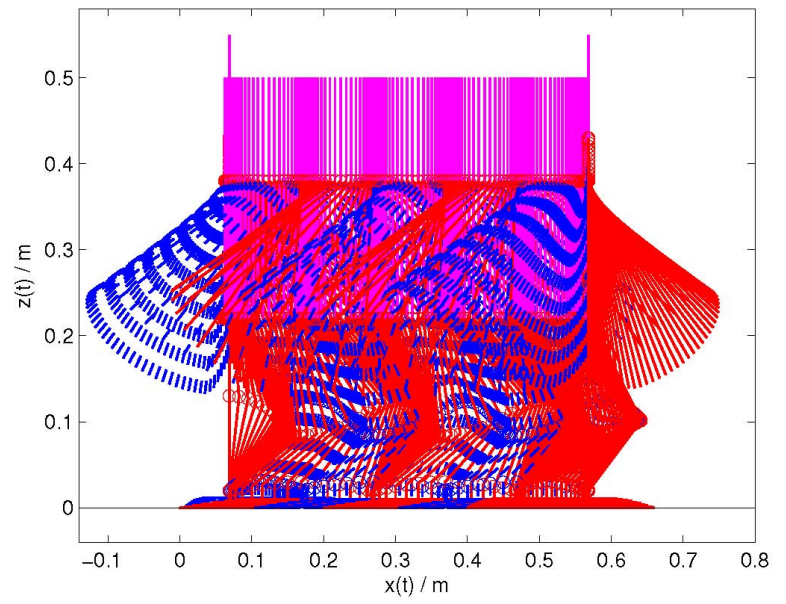

Fig. (5). The 2D schematic diagram of walking gait for humanoid robot.

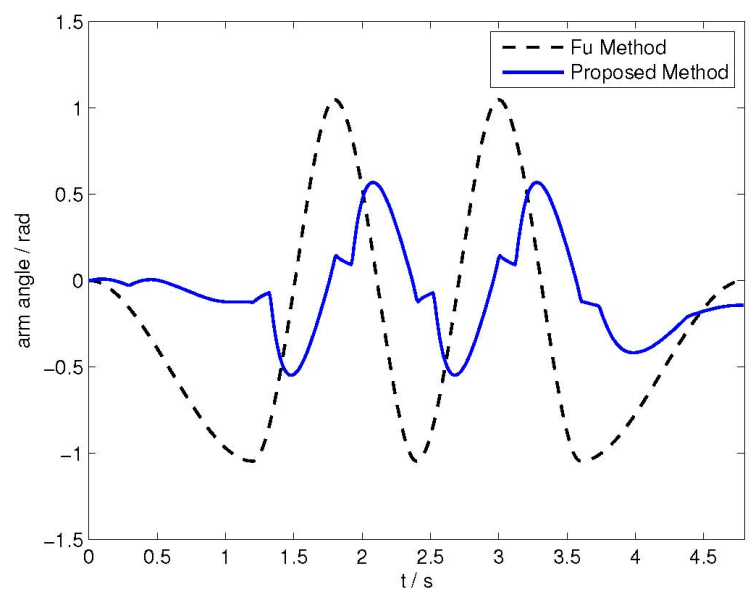

Fig. (6). Arm angle trajectories with different control methods.

are based on the gait proposed in FU [11]. In these figures, red, blue, pink sticks represent right foot/leg/arm, left foot/leg/arm and trunk, respectively.

In order to validate the effectiveness of the proposed method, the arm angle trajectories are provided in the comparison with the method in $\mathrm{Fu}$ [8], as shown in Fig. (6).
From the figures, the maximum angle degree descends $45 \%$ which dramatically save energy consumption. The arm awing angle trajectory is plotted in Fig. (7). Red and blue solid line depicts the tracking and desired trajectory respectively.

$\mathrm{Y}$-axis ZMP response trajectory is displayed in Fig. (8). In this figure, black solid and dashed lines depict maximum 


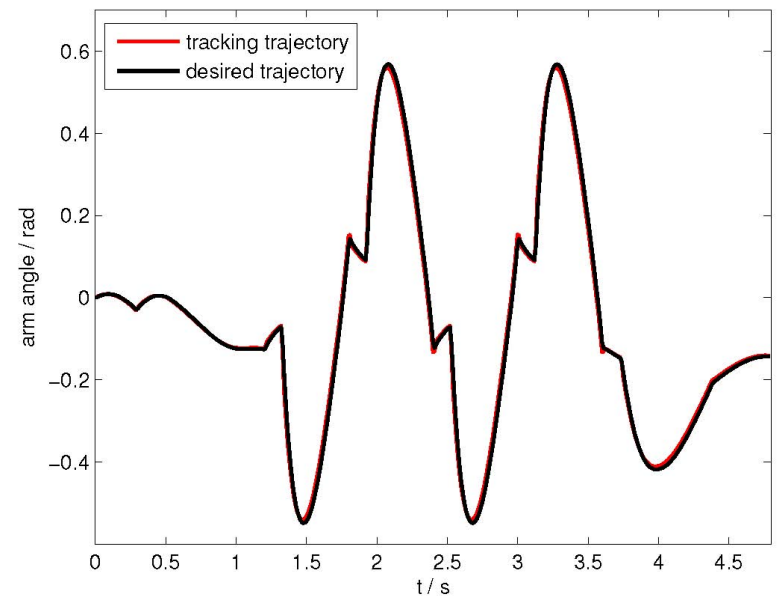

Fig. (7). Arm swinging angle trajectory.

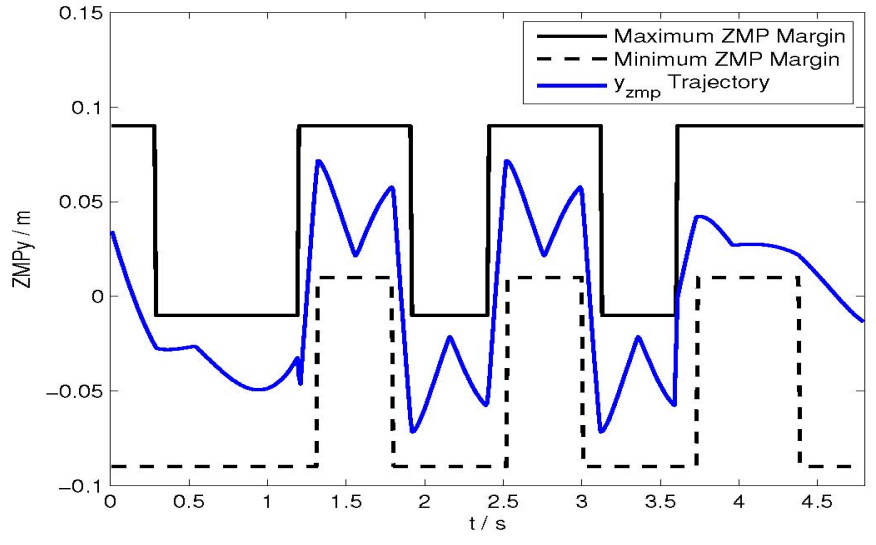

Fig. (8). y-axis ZMP trajectory using arms-swinging method.

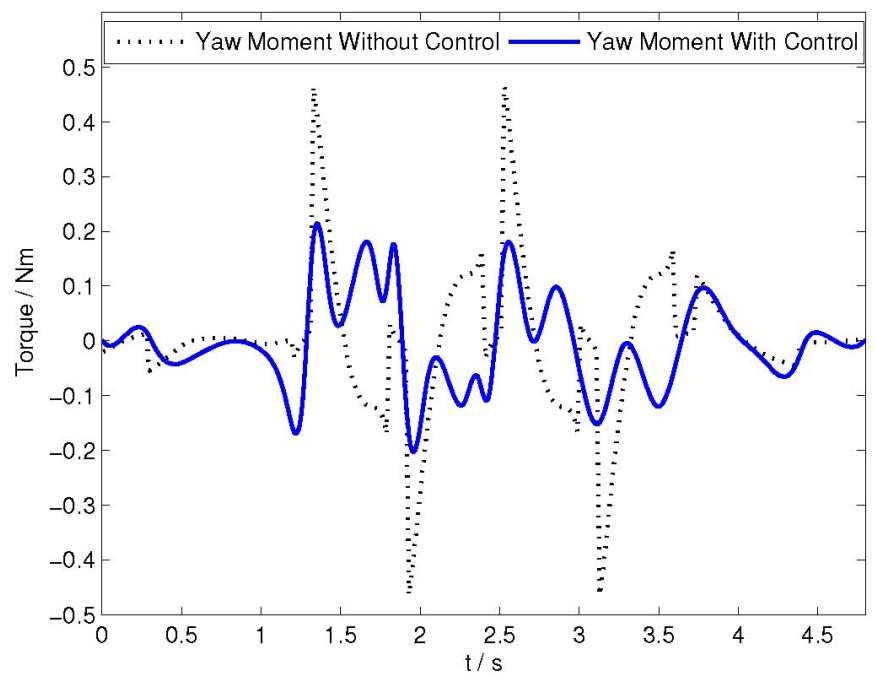

Fig. (9). Yaw moment with and without control.

and minimum ZMP boundary trajectories respectively and blue solid line symbolize y-axis ZMP response trajectory. Throughout the whole walking period, y-axis ZMP response is observed to be within the ZMP margin, indicating that dynamically-equilibrated walking cycles are obtained.
In Fig. (9), solid blue and dashed black lines indicate the yaw moment for with the proposed method and with the method in $\mathrm{Fu}$ [8], respectively. Using the method in this paper, the peak yaw moment response with is reduced for about $55 \backslash \%$ in comparison with $\mathrm{Fu}[8]$. 


\section{CONCLUSION}

In this paper, the problems of dynamic balance and energy consumption have been investigated. And a systematic way of generating arms-swinging trajectories is proposed to compensate the undesired yaw moment with the consideration of energy efficiency. By transferring the problem of the arms swinging trajectories optimization to quadratic programming problem, a parameter optimization method based on Lagrange multiplier rule is provided, which has a good computation performance. Several simulation experiments are conducted and the results validate the proposed method.

\section{CONFLICT OF INTEREST}

The authors confirm that this article content has no conflicts of interest.

\section{ACKNOWLEDGEMENTS}

This work is supported by National Nature Science Foundation under Grant (61300095, 61302115), funded by the Foundation for Distinguished Young Talents in Higher Education of Guangdong (2013LYM0104), supported by the Natural Science Foundation of Guangdong Province, China (S2013010015764, S2013010012307), supported by Foundation for Distinguished Young teachers in Higher Education of Guangdong, China (Yq2013204), funded by the Science and Technology Planning Program of Zhongshan City (2013A3FC0278, 2013A3FC0289), the PhD Start-up Fund of University of Electronic Science and Technology of China, Zhongshan Institute (413YKQ03) and the teaching quality engineering program of University of Electronic Science and Technology of China, Zhongshan Institute (ZLGC2012JY04).

\section{REFERENCES}

[1] D.P. Xing, and J.B. Su, "Arm/trunk motion generation for humanoid robot," Science in China, Series F: Information Sciences, vol. 53, no. 8, pp. 1603-1612, 2010.

[2] A. Dasgupta, and Y. Nakamura, "Making feasible walking motion of humanoid robots from human motion capture data," In: Proceedings on IEEE ICRA, Detroit, pp. 1044-1049, 1999.

[3] M. Vukobratovi, and B. Borovac, "Zero-moment point-thirty five years of its life," International Journal of Humanoid Robotics, vol. 1 , no. 01, pp. 157-173, 2004.

[4] M. Vukobratovi, B. Borovac, and V. Potkonjak, "ZMP: A review of some basic misunderstandings," International Journal of Humanoid Robotics, vol. 3, no. 02, pp. 153-175, 2006.

[5] W. Yu, G. Bao, Z. Wang, and W. Wu, "Fast walking pattern generation for humanoid robot using waist joint moment compensation,' Robot, vol. 32, no. 2, pp. 219-225, 2010.

[6] B. Ugurlu, J.A. Saglia, N.G. Tsagarakis, and D.G. Caldwell "Yaw moment compensation for bipedal robots via intrinsic angular momentum constraint," International Journal of Humanoid Robotics, vol. 9, no. 04, pp.1250033.1-1250033.27, 2012.

[7] J. Park, "Synthesis of natural arm swing motion in human bipedal walking," Journal of Biomechanics, vol. 41, no. 7, pp. 1417-1426, 2008.

[8] G.P. Fu, J.P. Chen, and Y.M. Yang, "A yaw moment counteracting method for humanoid robot based on arms swinging," Robot, vol. 34, no. 4, pp. 498-504, 2012.

[9] L.Y. Wang, Z. Liu, X.J. Zeng, and Y. Zhang "Gait control based on energy-efficiency optimization for biped robots," Control Theory and Application, vol. 28, no. 5, pp. 667-674, 2011.

[10] T. Hirabayashi, B. Ugurlu, A. Kawamura, and C. Zhu "Yaw moment compensation of biped fast walking using 3D inverted pendulum," In: International Workshop on Advanced Motion Control, Piscataway, NJ, USA: IEEE, 2008, pp. 296-300.

[11] G.P. Fu, Y.M. Yang, and C.L. Huang, "Walking pattern optimization based on particle swarm optimization for biped robot," Journal of Huazhong University of Science and Technology: Natural Science Edition, vol. 39, no. S2, pp. 355-358, 2011.

[12] Y.Y. Min, and Y.G. Liu, "Barbalat Lemma and its application in analysis of system stability," Journal of Shandong University (Engineering Science), vol. 37, no. 1. pp. 51-55, 2007.

(C) Yang and Deng; Licensee Bentham Open.

This is an open access article licensed under the terms of the Creative Commons Attribution Non-Commercial License (http://creativecommons.org/licenses/by-nc/3.0/) which permits unrestricted, non-commercial use, distribution and reproduction in any medium, provided the work is properly cited. 